\title{
Thermomechanical fatigue crack initiation in disc alloys using a damage approach
}

\author{
Daniel Leidermark ${ }^{1, *}$, Robert Eriksson ${ }^{1}$, James P. Rouse ${ }^{2}$, Christopher J. Hyde ${ }^{2}$, and Svjetlana Stekovic $^{3}$ \\ ${ }^{1}$ Division of Solid Mechanics, Linköping University, 58183 Linköping, Sweden \\ ${ }^{2}$ Gas Turbine and Transmission Research Centre, University of Nottingham, NG7 2RD, United Kingdom \\ ${ }^{3}$ Division of Engineering Materials, Linköping University, 58183 Linköping, Sweden
}

\begin{abstract}
A fatigue crack initiation model based on damage accumulation via a fatigue memory surface in conjunction with a plastic strain energy parameter was evaluated for thermomechanical fatigue loading in a gas turbine disc alloy. The accumulated damage in each hysteresis loop was summed up, and it was assumed that the damage at the stable state is repeated until failure occurs. Crack initiation occurs when enough fatigue damage has been obtained, and the number of cycles can thus be directly determined. The fatigue damage is highly coupled to the constitutive behaviour of the material, where the constitutive behaviour was modelled using a non-linear hardening description. Based on this, a stable state was achieved and the obtained damage could be extracted. A user-defined material subroutine was implemented, incorporating both the constitutive description and the fatigue damage accumulation. The framework was adopted in a finite element context to evaluate the thermomechanical fatigue crack initiation life of the disc alloy RR1000. From the evaluation it could be seen that a good prediction of the thermomechanical fatigue life was achieved compared to performed experiments.
\end{abstract}

\section{Introduction}

In the field of energy production and aircraft propulsion, with the use of gas turbines, thermomechanical fatigue (TMF) is one of the most damaging conditions that can possibly arise in a component. Within this area, focusing on high-pressure turbine disc alloys, the fatigue crack initiation life is of high interest, as well as the subsequent crack propagation. It has been observed that fatigue crack initiation is related to early surface damage, where extrusions and intrusions form sharp surface crack-like defects [1]. Thus, indicating that fatigue crack initiation is attributed to the surface and its evolution.

A traditional way of evaluating crack initiation life of a component is to perform a post-process operation of the results obtained in a finite element (FE) analysis. By extracting a set of internal variables at a stable state, number of cycles to crack initiation according to some defined life function that has been calibrated to experiments can be predicted. This life function can be some kind of Coffin-Manson [2,3] or Morrow [4] type of expression. One commonly used model to predict the fatigue crack initiation life is the Smith-Watson-Topper model [5]. It is an energy-based model that accounts for mean stress influence and assumes that damage is brought on by the maximum tensile stress. Another energy-density model that was used for low-cycle fatigue with dwell time at elevated temperatures is the model introduced by Ostergren [6]. In this model, damage is assumed to be caused by plastic deformation at tensile loading. Hence, the maximum tensile stress and the plastic strain range are used. It was shown by Kulawinski et al. [7] that both the Smith-Watson-Topper model and the Ostergren model were able to predict the TMF life with satisfactory results for Waspaloy, with a slightly better prediction using the Ostergren model. The model was further enhanced by combining the plastic stress-strain hysteresis with the initiation model by Zamrik and Renauld [8] and an Arrhenius term to yield even better correlation to the experiments.

An alternative approach, different to the post-processing method, is to incorporate a fatigue damage parameter in the FE-analysis, and accumulate damage until crack initiation occurs. Hence, closely linked to the constitutive model or directly implemented in a userdefined material model. One damage approach, proposed for high-cycle fatigue loading is the one proposed by Ottosen et al. [9], which is based on the load history by defining an endurance surface. The endurance surface moves with the loading using a "back-stress" term. A continuum damage approach based on the evolution of the associated endurance function defines the acquired damage, which eventually gives failure. Another fatigue damage model was proposed by Jiang [10]. It uses a plastic strain energy damage parameter coupled to a critical-plane [11] with a material memory surface to

Corresponding author: daniel.leidermark@,liu.se 
model fatigue crack initiation behaviour under multiaxial loading.

The aim of this paper is to define and evaluate a crack initiation model based on a damage accumulation approach and to predict the TMF crack initiation life from performed experiments of the turbine disc alloy RR1000. An engine relevant out-of-phase TMF loading cycle was considered.

\section{Experiments}

The studied material in question is the coarse grain powder processed $\gamma$ ' strengthened polycrystalline nickelbase turbine disc alloy RR1000. The chemical composition of the material, with decreasing weight percentage, is Ni-18.5Co-15Cr-5Mo-3.6Ti-3Al-2Ta$0.5 \mathrm{Hf}-0.06 \mathrm{Zr}-0.027 \mathrm{C}-0.015 \mathrm{~B}$. The manufacturing process steps and succeeding heat treatments of the material are given in Li et al. [12].

Five round, as-machined, specimens were tested at engine relevant conditions. The test specimens were machined from the pancake forging to a diameter of approximately $7.6 \mathrm{~mm}$. The applied engine relevant load cycle is a type of out-of-phase strain-controlled TMF cycle containing a hold-time of 30 s. The temperature was cycled between $300-675^{\circ} \mathrm{C}$ and different mechanical strain ranges $\left(\Delta \varepsilon_{\text {mech }}\right)$ were applied. The tests were performed in a hydraulic testing machine using induction heating and the strain ranges were controlled by a hightemperature extensometer with a gauge length of 12.75 $\mathrm{mm}$. The number of cycles to TMF crack initiation $\left(N_{i}\right)$ was determined by a $10 \%$ load-drop of the maximum tensile stress obtained in each cycle. The received crack initiation lives have been normalised with respect to the longest life obtained in the experiments due to confidentiality issues, see Table 1 for the experimental data.

\section{Modelling}

\subsection{Constitutive model}

For the purpose of describing the constitutive behaviour of the disc alloy in question, a rate dependent Perzyna viscoplastic material model [13] was implemented as a user-defined material subroutine in an FE-context. The constitutive model is able to describe the cyclic evolution of the material by two non-linear ArmstrongFrederick back-stress terms [14] and a linear as well as a saturated isotropic hardening term. In what follows, all tensors are presented in index notation, where secondorder tensors are quantified by upper-case Roman or Greek-letters

and

Table 1. Experimental TMF data of the round specimens.

\begin{tabular}{cccc}
\hline Specimen & $\begin{array}{c}\text { Diameter } \\
{[\mathrm{mm}]}\end{array}$ & $\Delta \varepsilon_{\text {mech }}[\%]$ & $\begin{array}{c}\text { Normalised } \\
N_{i}\end{array}$ \\
\hline
\end{tabular}

\begin{tabular}{llll}
\hline$S 1$ & 7.61 & 0.8512 & 0.695 \\
$S 2$ & 7.60 & 0.8976 & 0.526 \\
$S 3$ & 7.62 & 0.8521 & 1.000 \\
$S 4$ & 7.60 & 0.8984 & 0.593 \\
$S 5$ & 7.61 & 0.8803 & 0.604 \\
\hline
\end{tabular}

scalar valued parameters are defined by lower-case Roman and Greek-letters. The following yield function was employed

$$
f=\sigma_{e q}^{v M}\left(\hat{\sigma}_{i j}-B_{i j}\right)-r-\sigma_{Y}
$$

where $\hat{\sigma}_{i j}$ is the deviatoric stress tensor defined as $\hat{\sigma}_{i j}=\sigma_{i j}-\frac{1}{3} \sigma_{k k} \delta_{i j}, \delta_{i j}$ is the Kronecker's delta and $B_{i j}$ is the total back-stress tensor. Isotropic hardening is described by $r, \sigma_{Y}$ is the initial yield limit and $\sigma_{e q}^{v M}$ is the von Mises equivalent stress, defined as

$$
\sigma_{e q}^{v M}=\sqrt{\frac{3}{2}\left(\hat{\sigma}_{i j}-B_{i j}\right)\left(\hat{\sigma}_{i j}-B_{i j}\right)}
$$

The total back-stress term is based on additively decomposition [15] of the two individual back-stress terms, $B_{i j}=\sum_{k} B_{i j}^{k}$, where the evolution law for each term is defined by

$$
\dot{B}_{i j}^{k}=c_{k}\left(\frac{2}{3} a_{k} \dot{\varepsilon}_{i j}^{v p}-B_{i j}^{k} \dot{\lambda}\right), k=1,2
$$

with the individual material parameters $c_{k}$ and $a_{k}$. To correlate the different stages of the cyclic evolution of the stress state, two isotropic hardening terms have been adopted; one linear and one saturated. These are summed up to the total isotropic hardening term $\left(r=r_{1}+r_{2}\right)$, in which the respective evolution law is given by

$$
\begin{gathered}
\dot{r}_{1}=q_{1} \dot{\lambda} \\
\dot{r}_{2}=h\left(q_{2}-r_{2}\right) \dot{\lambda}
\end{gathered}
$$

where $q_{1}$ describes the long term cyclic softening and the rapid initial softening is accounted for by $h$ and $q_{2}$, which describes the rate of cyclic softening and the saturation.

The evolution law of the viscoplastic strain tensor is defined by the Perzyna flow rule based on the adopted yield function as

$$
\dot{\varepsilon}_{i j}^{v p}= \begin{cases}0 & , f \leq 0 \\ \dot{\lambda} \frac{\partial f}{\partial \sigma_{i j}} & , f>0\end{cases}
$$

in which the viscoplastic multiplier is defined by the following power-law relation

$$
\dot{\lambda}=\left(\frac{f}{\eta}\right)^{m}
$$


where $m$ and $\eta$ are material parameters accounting for the viscous effects.

Material parameters for the above presented constitutive model and material were acquired from an optimization procedure based on stress partitioning and cycle jumping. An in-depth description of this procedure can be found in Rouse et al. [16], as well as generated material parameters. The parameters are temperature dependent and are defined continuous over the evaluated temperature range in the engine relevant TMF cycle.

\subsection{Fatigue damage model}

As crack initiation is mainly a surface dominant phenomena, the surface damage has to be evaluated. Hence, a fatigue damage model based on the memory surface concept [10] was used to evaluate the crack initiation life. The model consist of a fatigue damage term that incorporates the material memory and plastic strain energy in the point of interest, hence eliminating the need of a cycle-counting method.

In analogy with the yield surface in plasticity, a fatigue memory surface can be defined as

$$
g=\sqrt{\hat{\sigma}_{i j} \hat{\sigma}_{i j}}-\sqrt{\frac{2}{3} \sigma_{m e m}} \leq 0
$$

where $\sigma_{\text {mem }}$ is the memory stress, which initially is set to the fatigue endurance limit of the material, $\sigma_{\text {end }}$. The evolution law of the memory stress is defined according to

$$
\begin{aligned}
& \dot{\sigma}_{m e m}=\sqrt{\frac{3}{2} H(g)\left\langle\frac{\hat{\sigma}_{i j} \dot{\hat{\sigma}}_{i j}}{\sqrt{\hat{\sigma}_{i j} \hat{\sigma}_{i j}}}\right\rangle} \\
& -\beta(1-H(g))\left(\sigma_{m e m}-\sigma_{\text {end }}\right) \dot{\lambda}
\end{aligned}
$$

The Heaviside function, $H(g)$, makes sure that the memory surface contracts when the stress state is lower than the memory stress $(g<0)$ and expands when it is on the memory surface $(g=0)$. The parameter $\beta$ defines how much the surface contracts, and $\langle\cdot\rangle$ is the Macaulay bracket.

The damage accumulation is then directly coupled to the memory surface, using the memory stress to account for load-sequence effects. For proportional loading conditions the evolution of the damage is thus described by

$$
\dot{\omega}=\left\langle\sigma_{m e m}-\sigma_{e n d}\right\rangle^{\alpha} \dot{\psi}^{p}
$$

where $\alpha$ is a material parameter and $\psi^{p}$ is the plastic strain energy.

A simple multiaxial approach has been adopted for the plastic strain energy evolution law with inspiration of the work by Ostergren [6]

$$
\dot{\psi}^{p}=H\left(\sigma_{k k}\right) \sigma_{e q}^{v M} \dot{\lambda}
$$

where $H\left(\sigma_{k k}\right)$ accounts for micro-crack closure by making sure that damage is only accumulated during the tensile part from the loading cycle.

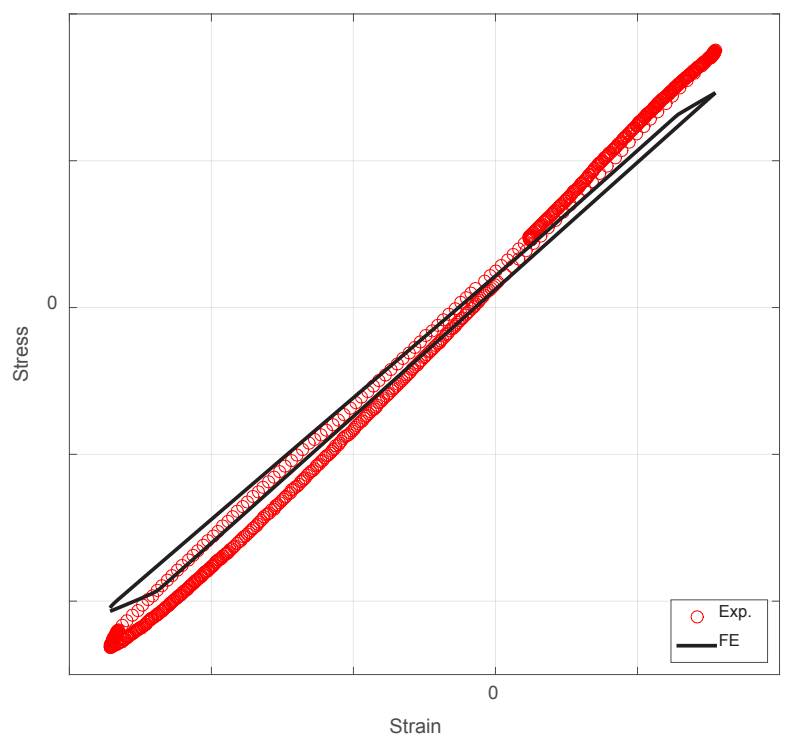

Figure 1. The stable experimental and simulated hysteresis loop of $S 1$.

It have to be pointed out that the fatigue damage model for crack initiation is not coupled to the stress update in the constitutive model, as traditionally done for continuum damage models. The fatigue damage model is a stand-alone model with inputs directly from the constitutive model, as it is implemented as a subroutine within the above described user-defined material subroutine. Hence, the stress and strain state in each increment is directly feed into the fatigue damage subroutine. Thus, the history of the damage is accounted for and evolves in the subsequent iterations until the critical damage is reached, initiating a macroscopic crack. Consequently, the damage accumulation during each load cycle is summed up to the critical value $\omega_{c}$, representing crack initiation. This is quantified as

$$
\sum_{i=1}^{N_{i}} \Delta \omega_{i}=\omega_{c}
$$

where $\Delta \omega_{i}$ is the damage accumulated during a load cycle.

\section{Evaluation and calibration of TMF crack initiation}

A quarter of the specimen was modelled and analysed, using only the straight part over the extensometer gauge length. This was due to that the material in question is isotropic and aspects in applying the boundary conditions to achieve the correct $\Delta \varepsilon_{\text {mech }}$ in the TMF 
cycle. The FE-model consists of 429 fully integrated 8noded brick elements. Symmetry boundary conditions as well as locking in the axial direction at the bottom and loading at the top, according to the engine relevant TMF cycle, were applied. FE-simulations were performed using the FE-software LS-DYNA [17], version R7, with the user-defined material-fatigue model. Figure 1 shows an example of an obtained stable hysteresis loop from the

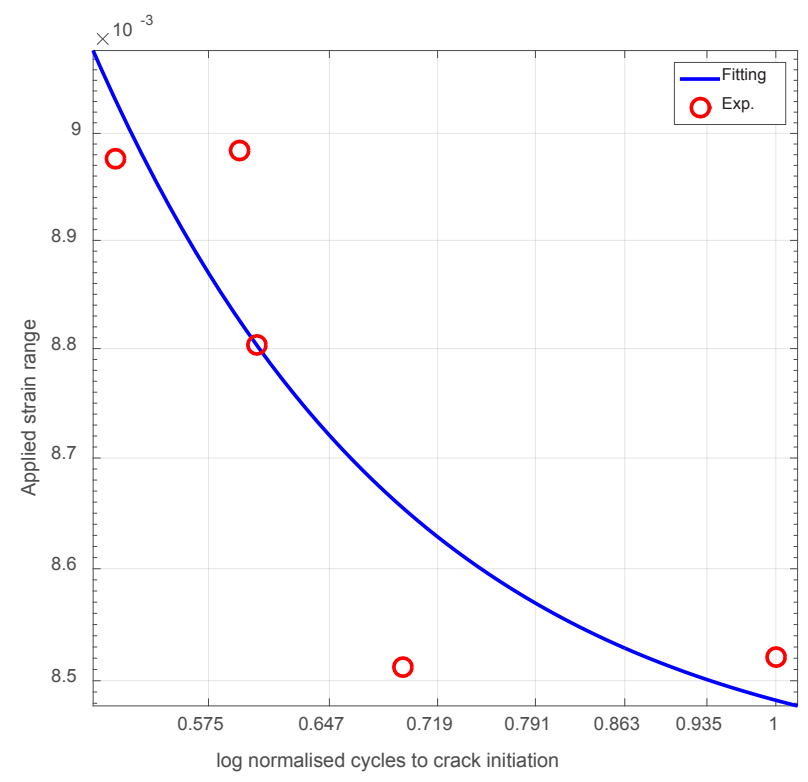

Figure 2. Applied strain range versus number of cycles to crack initiation.

FE-simulation compared to the experimental one, displaying the response of the $S 1$ specimen.

The fatigue endurance limit is present in the fatigue damage model, and this value can be taken from the Wöhler curve at infinite life (typically $10^{6}$ cycles to failure) for the material in question. However, due to limiting resources of data for the present material, only rough estimates of the endurance limit at certain temperatures were available. Hence, instead of using these uncertain values, an evaluation of the endurance limit for the employed TMF cycle was determined based on the procedure suggested by Jiang [10]. Ranges were used rather than amplitudes due to the applied unsymmetrical TMF load cycle. The applied strain ranges were plotted against the number of cycles to crack initiation, see Figure 2, and a curve fit was performed using the following equation

$$
\left(\Delta \varepsilon_{\text {mech }}-\Delta \varepsilon_{\text {end }}\right)^{d} N_{i}=e
$$

where $\Delta \varepsilon_{\text {mech }}$ and $N_{i}$ are taken from Table 1 and $\Delta \varepsilon_{\text {end }}$, $d$ and $e$ are fitting parameters. One can interpret $\Delta \varepsilon_{\text {end }}$ as the endurance limit in strain range. Then, the generated $\Delta \varepsilon_{\text {end }}$ value was compared to the cyclic stress-strain curve to obtain the corresponding endurance limit of the material for this specific TMF cycle. This was done by evaluating the experimental Ramberg-Osgood relationship [18], hence the following expression was used

$$
\frac{\Delta \varepsilon}{2}=\frac{\Delta \sigma}{2 E}+\left(\frac{\Delta \sigma}{2 K}\right)^{1 / n}
$$

where $K$ and $n$ are material parameters based on the experiments. These two parameters were evaluated by fitting Equation (14) to the stress and strain ranges of the

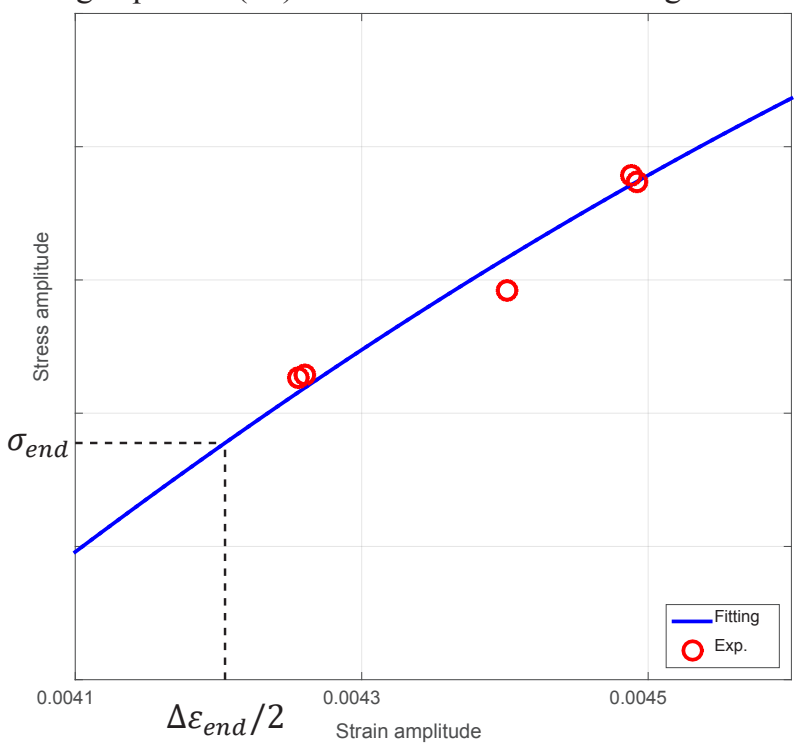

Figure 3. The Ramberg-Osgood curve and evaluation of the endurance limit.

performed experiments, and from this relationship the endurance limit $\sigma_{\text {end }}$ could be determined, see Figure 3. The used Young's modulus $E$ in the expression was taken as the experimental mean modulus, with regard to the temperature, during the on-loading in the TMF cycle.

Based on that only constant amplitude loading is present in the applied TMF load cycle, the parameter $\beta$ was set equal to zero based on the work of Jiang [10]. This parameter is used for contracting the memory surface when going from a large amplitude to a lower in the succeeding cycle, hence changing the memory stress, and as no such loading is present in the load cycle no contribution will be obtained.

The critical damage was determined by post-processing the performed FE-simulations, in which the accumulated fatigue damage during each cycle was extracted in an element far away from the applied boundary conditions at the surface of the specimen. The extraction is only necessary up to the cycle in which a stable state is obtained, hence a simplification of the expression in Equation (12) was performed according to

$$
\omega_{c}=\Delta \omega_{1}+\cdots+\Delta \omega_{x}+\Delta \omega_{\text {stable }}\left(N_{i}-x\right)
$$

where a stable state is reached after $x$ number of cycles. In this manner, not all cycles up to the "real" crack initiation cycle need to be simulated in the FE-context, hence saving computational time. A difference of less 
than $5 \%$ between the accumulated fatigue damage from the previous cycle was quantified as a stable state. From this, the determination of the critical damage was done under the assumption that the value of $\alpha$ is known, which was not the case initially. Thus, a calibration involving $\alpha$ and the critical fatigue damage was conducted, by use of a best praxis fitting procedure based on the experimental results and FE-simulations. As the critical fatigue damage is to

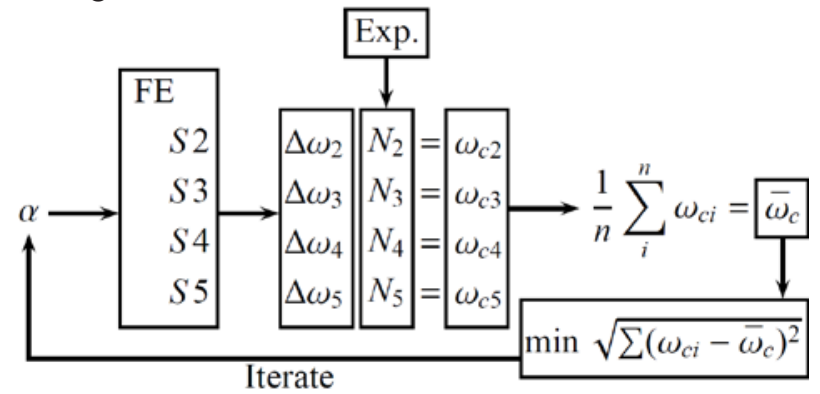

Figure 4. The best praxis fitting of the fatigue damage parameters.

be equal for any applied strain range in the material, a minimisation of the error with respect to the mean value of the critical fatigue damage for a given $\alpha$ value from each of the specimens was used, utilising Equation (15). Hence, both $\alpha$ and $\omega_{c}$ were obtained by coupling the experimental crack initiations lives with the fatigue damage model. A schematic display of the procedure is depict in Figure 4, where responses from $S 2, S 3, S 4$ and $S 5$ were used in the calibration and $S 1$ was used to verify the crack initiation model. The iterative procedure was stopped when an optimal solution of the comparable difference in the critical fatigue damage was reached, and a final mean value of these was used as the critical fatigue damage.

Based on the above described fitting procedure, $\alpha$ and $\omega_{c}$ could be determined. An FE-analysis of $S 1$ with the obtained values was performed to verify the adopted fatigue damage model. This can be seen in Figure 5, where the normalised predicted TMF crack initiation lives in the FE-context are compared to the ones obtained in the experiments. To access the quality of the model, a scatter band based on the standard deviation in the predicted lives from the specimens used in the calibration with respect to the experiments was added in the figure. One can see that approximately all calibrated specimens lie within the standard deviation and that the verifying specimen $S 1$ are captured within a factor of five standard deviations, corresponding to a factor of 1.3707 to the experimental TMF crack initiation life. The normalised predicted TMF crack initiation lives of the specimens and the relative factor between the predicted and experimental lives are summarised in Table 2.

\section{Discussion}

From the analyses it could be observed that the adopted fatigue damage model correlate well with the observed experimental behaviour, see Figure 5 . As can be seen in Table 1, there is a noticeable different for the experimental lives between $S 1$ and $S 3$. This gives a small misguidance in the calibration procedure of the fatigue parameters, as only $S 3$ is included. Hence, focus is given to the longer life at the comparable strain range level, which in hand yields a larger relative factor in the predicted TMF crack initiation life of S1, cf. Table 2. Thus, more performed tests would level out these kind of deviating results. Furthermore, from the results displayed

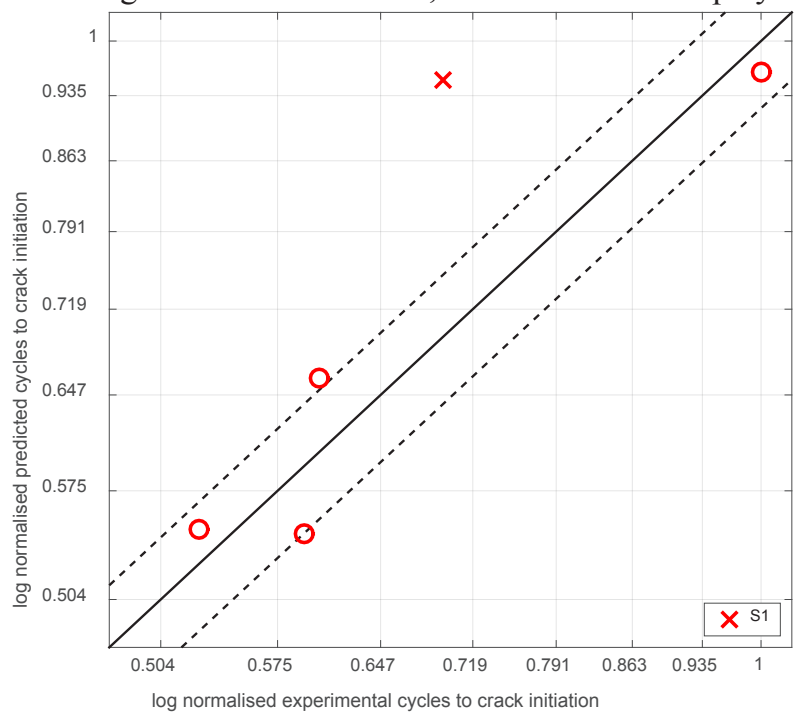

Figure 5. The normalised predicted TMF crack initiation lives versus the experimental ones.

in Figure 5 it can be seen that all the predicted crack initiation lives from the FE-analyses lie within a factor of 1.3707 from the experiments, corresponding to approximately five standard deviations from the calibrated set of specimens, where the verifying analysis of $S 1$ is the one setting this factor. This gives a sound interpretation that the proposed fatigue damage model enables to capture the correct behaviour of the investigated material under the applied engine relevant load conditions.

It is to be noted that a difference in Young's modulus is present in Figure 1 between the experimental curve and the one obtained from the FE-simulation. A reason is due to different material batches, as the identified material parameters in Rouse et al. [16] comes from a specific batch which is different from the one used in the evaluated TMF experiments. It is common that scatter in material properties are present between different batches of the same material. This is due to that different sizes of pancake forgings have been used, which were affected by the heat treatment, and as a consequence difference in grain size, grain size distribution, $\gamma^{\prime}$ size and distribution were obtained. However, the plastic strain range of the two hysteresis loops are approximately the same, which is important from a fatigue perspective as the fatigue damage model is based on the plastic strain energy. Furthermore, the difference in Young's modulus generates a little lower maximum and minimum stresses compared to the experiment. 
Table 2. The predicted TMF crack initiation lives and the relative factor with respect to the experimental lives.

\begin{tabular}{ccc}
\hline Specimen & Normalised predicted $N_{i}$ & Factor \\
\hline$S 1$ & 0.9528 & 1.3707 \\
$S 2$ & 0.5490 & 1.0440 \\
$S 3$ & 0.9622 & 0.9622 \\
$S 4$ & 0.5459 & 0.9200 \\
$S 5$ & 0.6610 & 1.0952 \\
\hline
\end{tabular}

The proposed method to evaluate the TMF crack initiation life is based on that the fatigue damage is extracted until a stable state is reach (5\% difference) and then using the last value to predict the life with the critical damage, $c f$. Equation (15). This is a very straightforward, simple and computationally easy approach. On the other hand, this approach might not yield an entirely correct behaviour of the fatigue damage process, as the obtained damage will likely be further reduced with successive cycles and eventually reach a steady-state condition with no difference in damage. To capture this, a cycle-by-cycle FE-simulation has to be performed up to the cycle in which the critical damage is obtained by using Equation (12). This is a rather computational heavy procedure as many cycles need to be performed. Hence, to accelerate the simulation effort a cycle jumping procedure can be adopted, see e.g. Kontermann et al. [19], where the internal variables (including the memory stress) are extrapolated a number cycles forward. Though, one need to keep in mind that a cycle jumping procedure is in it self a complex element, and adoption of which might end up in a more complicated approach than the herein proposed simple method.

The performed experiments were conducted on smooth specimens, where a uniaxial state is present. This simplifies matters, as a uniaxial response is obtained in the FE-analyses and a uniaxial lifing criterion could have been adopted. As real component geometry contain stress-raising features, such as notches and holes, a multiaxial lifing criterion have to be used, which is the case in this work by utilising the von Mises equivalent stress in Equation (11). Hence, from a multiaxial perspective the implemented fatigue damage model can directly be utilised. The choice of the plastic strain energy parameter will likely change the level of fatigue damage, where e.g. a critical-plane assumption [11] can be adopted instead of the above simple multiaxial approach. Further development of the fatigue damage model can be to include a multiaxial critical-plane assumption, where the stress and strain are evaluated on a plane at e.g. a notched feature. This plane can also be used for the subsequent crack growth.

The herein defined and adopted endurance limit is not temperature dependent, as it is based on the applied engine relevant TMF load cycle. This means that the defined endurance limit is viewed as an approximate endurance limit with respect to the applied temperature field in this specific load cycle. One can of course reason that a temperature dependent endurance limit based on Wöhler curves is to be preferred, but due to lack of data this could not be done. By using the defined endurance limit, obtained from the Ramberg-Osgood relation, a slight difference compared to a temperature dependent limit will be generated within the fatigue damage accumulation, as the temperature difference of $375^{\circ} \mathrm{C}$ is present during the loading cycle. Another way, could have been to apply a reduction factor based on the temperature. Additionally, one could have used the temperature dependent ultimate tensile strength and scale it with a suitable factor to define the endurance limit.

Uncertainties are always present in modelling work, as each simplification adds up and are displayed in the final response. Based on the performed work, only five test specimens were used in evaluation of the fatigue damage model. This is not enough tests to generate a statistical good response, as one or two specimens might generate a TMF crack initiation life which are not entirely correct due to some uncontrollable factors occurring during the experiments, $c f$. $S 1$ and $S 3$. Another uncertainty lies in the used material parameters, both for the constitutive model as well as the fatigue model. These are crucial for the final response, as a small variation will generate large repercussions in the results. As mentioned above, regarding the uncertainties in using different material batches, a difference in the simulated hysteresis loop will of course contribute to a discrepancy compared to the experimental one, and as a consequence a discrepancy will be obtained in number of cycles to crack initiation. Based on this, a large statistical foundation is needed to correctly evaluate the fatigue lives.

Disregarding the material parameters and different responses due to material batches, the constitutive model is what drives the fatigue damage model. The better conformance of the constitutive behaviour a more accurate TMF crack initiation life prediction will be generated. This, as the fatigue damage model is based on the responses obtained locally in the FE-model from the constitutive model.

\section{Conclusions}

The main conclusions that can be drawn from the presented work are:

- A satisfactory response of the constitutive behaviour compared to the experiments was obtained. The small difference is due to that a different material batch was used to calibrate the constitutive model.

- The adopted fatigue damage model predicts the TMF crack initiation lives within a factor of 1.3707 compared to experiments.

- The fatigue damage model facilitates a simple approach for accounting for load-sequence effects and eliminates cycle-counting methods.

- Further experiments need to be performed to reduce statistical uncertainties that can be drawn from the present work, as only four specimens were used in the calibration of the fatigue damage model. Hence, 
minimizing the discrepancy in TMF crack initiation life for the verifying specimen.

The study has received funding from the Clean Sky 2 Joint Undertaking under the European Union's Horizon 2020 research and innovation programme under grant agreement No 686600 .

\section{References}

[1] Polák J., Man J., Procedia Engineering 101, 386394 (2015)

[2] Tavernelli J.F., Coffin L.F., Journal of Basic Engineering 84, 533-537 (1962)

[3] Manson S.S., Journal of Basic Engineering 84, 537-541 (1962)

[4] Socie D.F., Morrow J.D., Risk and Failure Analysis for Improved Performance and Reliability, 141-194 (1980)

[5] Smith R.N., Watson P., Topper T.H., Journal of Materials 5, 767-778 (1970)

[6] Ostergren W.J., Journal of Testing and Evaluation 4, 327-339 (1976)

[7] Kulawinski D., Weidner A., Henkel S., Biermann H., International Journal of Fatigue 81, 21-36 (2015)

[8] Zamrik S.Y., Renauld M.L., Thermo-mechanical Fatigue Behavior of Materials STP 1371 ASTM, 119-137 (2000)

[9] Ottosen N.S., Stenström R., Ristinmaa M., International Journal of Fatigue 30, 996-1006 (2008)

[10] Jiang Y., Fatigue \& Fracture of Engineering Materials \& Structures 23, 19-32 (2000)

[11] Karolczuk A., Macha E., International Journal of Fracture 134, 267-304 (2005)

[12] Li H.Y., Sun J.F., Hardy M.C., Evans H.E., Williams S.J, Doel T.J.A., Bowen P., Acta Materialia 90, 355-369 (2015)

[13] Perzyna P., Advances in Applied Mechanics 11, 313-354 (1971)

[14] Frederick C.O., Armstrong P.J., Materials at High Temperatures 24, 1-26 (2007)

[15] Chacboche J.L., International Journal of Plasticity 5, 247-302 (1989)

[16] Rouse J.P., Hyde C.J., Leidermark D., Stekovic S., Jones J., Whittaker M., Lancaster R., Pattison S., Grant B., Fatigue 2018, MATEC Web of Conferences (2018)

[17] Hallquist J.O., LS-DYNA Theory Manual (Livermore Software Technology Corporation, Livermore, 2006) 680

[18] Ramberg W., Osgood W.R., Technical Notes National Advisory Committee for Aeronautics 902, 1-29 (1943)

[19] Kontermann C., Scholz A., Oechsner M., Materials at High Temperatures 31, 334-342 (2014) 